\title{
Quantum Spin Liquids in Frustrated Spin-1 Diamond Antiferromagnets
}

\author{
Finn Lasse Buessen, ${ }^{1}$ Max Hering, ${ }^{2}$ Johannes Reuther, ${ }^{2,3}$ and Simon Trebst ${ }^{1}$ \\ ${ }^{1}$ Institute for Theoretical Physics, University of Cologne, 50937 Cologne, Germany \\ ${ }^{2}$ Dahlem Center for Complex Quantum Systems and Institut für Theoretische Physik, Freie Universität Berlin, \\ Arnimallee 14, 14195 Berlin, Germany \\ ${ }^{3}$ Helmholtz-Zentrum Berlin für Materialien und Energie, Hahn-Meitner-Platz 1, 14019 Berlin, Germany
}

(Received 28 June 2017; revised manuscript received 21 September 2017; published 29 January 2018)

\begin{abstract}
Motivated by the recent synthesis of the spin- $1 A$-site spinel $\mathrm{NiRh}_{2} \mathrm{O}_{4}$, we investigate the classical to quantum crossover of a frustrated $J_{1}-J_{2}$ Heisenberg model on the diamond lattice upon varying the spin length $S$. Applying a recently developed pseudospin functional renormalization group approach for arbitrary spin- $S$ magnets, we find that systems with $S \geq 3 / 2$ reside in the classical regime, where the lowtemperature physics is dominated by the formation of coplanar spirals and a thermal (order-by-disorder) transition. For smaller local moments $S=1$ or $S=1 / 2$, we find that the system evades a thermal ordering transition and forms a quantum spiral spin liquid where the fluctuations are restricted to characteristic momentum-space surfaces. For the tetragonal phase of $\mathrm{NiRh}_{2} \mathrm{O}_{4}$, a modified $J_{1}-J_{2}^{-}-J \frac{\perp}{2}$ exchange model is found to favor a conventionally ordered Néel state (for arbitrary spin $S$ ), even in the presence of a strong local single-ion spin anisotropy, and it requires additional sources of frustration to explain the experimentally observed absence of a thermal ordering transition.
\end{abstract}

DOI: 10.1103/PhysRevLett.120.057201

In the field of frustrated magnetism, spinel compounds of the form $A B_{2} X_{4}$ (with $X=\mathrm{O}, \mathrm{Se}, \mathrm{S}$ ) have long been appreciated as a source of novel physical phenomena [1]. $B$-site spinels with magnetic $B$ ions and nonmagnetic $A$ ions, such as $A \mathrm{Cr}_{2} \mathrm{O}_{4}$ or $A \mathrm{~V}_{2} \mathrm{O}_{4}$ (with $A=\mathrm{Mg}, \mathrm{Zn}, \mathrm{Cd}$ ), realize pyrochlore antiferromagnets where geometric frustration manifests itself in a vastly suppressed ordering temperature relative to the Curie-Weiss temperature. Conceptually, the pyrochlore Heisenberg antiferromagnet is a paradigmatic example of a three-dimensional spin liquid [2,3], in both its classical [4,5] and quantum [6,7] variants. $A$-site spinels, with nonmagnetic $B$ ions and magnetic $A$ ions forming a diamond lattice, have caught broader attention some ten years ago with the synthesis of $\mathrm{MnSc}_{2} \mathrm{~S}_{4}$ [8], $\mathrm{FeSc}_{2} \mathrm{~S}_{4}$ [8], and $\mathrm{CoAl}_{2} \mathrm{O}_{4}$ [9,10] that, similar to the $B$-site spinels, exhibit a dramatic suppression of their ordering temperature. At first sight counterintuitive due to the unfrustrated nature of the diamond lattice, it was conceptualized [11] that a sizable next-nearest neighbor coupling (connecting spins on the fcc sublattices of the diamond lattice) induces strong geometric frustration. Indeed, it could be shown that the classical Heisenberg model with both nearest and next-nearest neighbor exchange

$$
\mathcal{H}=J_{1} \sum_{\langle i, j\rangle} \mathbf{S}_{i} \mathbf{S}_{j}+J_{2} \sum_{\langle i, j\rangle\rangle} \mathbf{S}_{i} \mathbf{S}_{j}
$$

exhibits highly-degenerate coplanar spin spiral ground states for antiferromagnetic $J_{2}>\left|J_{1}\right| / 8$. Describing a single coplanar spin spiral by a momentum vector $\vec{q}$ (indicating its direction and pitch), the degenerate ground-state manifold can be captured by a set of $\vec{q}$ vectors that span a "spin spiral surface" in momentum space [11] as illustrated in Fig. 1. While these spiral surfaces bear a striking resemblance to Fermi surfaces [12], they are considerably more delicate objects that can be easily destroyed by small perturbations to the Hamiltonian (1) (such as further interactions) or even by fluctuations $[11,13]$ that will induce an order-by-disorder transition into a simple magnetically ordered state (typically captured by a single $\vec{q}$ vector). Such a description of the magnetism of $A$-site spinels in terms of classical local moments has
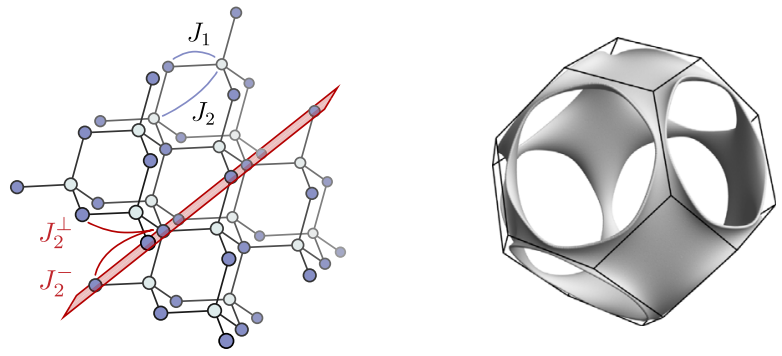

FIG. 1. Frustrated diamond lattice antiferromagnet. Left: Diamond lattice with nearest $\left(J_{1}\right)$ and next-nearest neighbor coupling $\left(J_{2}\right)$. A tetragonal distortion of the lattice along one spatial axis (orthogonal to the plane indicated in red) splits the 12 next-nearest neighbor couplings into a group of 4 in-plane terms $\left(J_{2}^{-}\right)$and 8 out-of-plane terms $\left(J_{2}^{\perp}\right)$. Right: Spin spiral surface for $J_{2} /\left|J_{1}\right|=0.73$ plotted in the first Brillouin zone (solid lines). 
proved sufficient to capture the physics of the $\mathrm{Mn}$ and Co-based spinels [11,14-16] with local moments $S=5 / 2$ and $S=3 / 2$, respectively, while the physics of $\mathrm{FeSc}_{2} \mathrm{~S}_{4}$ $(S=2)$ is dominated by the formation of a spin-orbit coupled local moment $[17,18]$.

Earlier this year, the synthesis of the first spin- $1 A$-site spinel has been reported- $\mathrm{NiRh}_{2} \mathrm{O}_{4}$, which is found to exhibit no thermal ordering transition down to $0.1 \mathrm{~K}$ [19], possibly indicating the formation of a quantum spin liquid ground state. This motivates us to consider the quantum version of the minimal exchange model (1) for spins of arbitrary length $S$ in this manuscript and ask whether qualitatively new physics arises in the crossover from the classical to the quantum regime (upon decreasing the spin length). We work with a pseudofermion functional renormalization group (PF-FRG) approach [20] that has been proven capable of handling competing interactions and emergent spin liquid physics in three-dimensional, frustrated quantum magnets [21-23], and which has recently been generalized to spin- $S$ systems [24]. Our numerical results indicate that a distinct classical to quantum crossover occurs for spin $S=3 / 2$. While the low temperature physics is dominated, independent of the spin length $S$, by the formation of spin spiral correlations that manifest themselves in the spin structure factor in the form of clearly discernible spin spiral surfaces (akin to the one shown in the right panel of Fig. 1), we find that only for systems with spin $S \geq 2$ do these correlations proliferate and give rise to a thermal phase transition into a magnetically ordered ground state. For systems with spin $S \leq 1$, we find no indication of a thermal phase transition for the full extent of the spiral regime $J_{2} / J_{1}>1 / 8$. The system with $S=3 / 2$ is found to sit precisely at the border with no thermal phase transition occurring in the regime $1 / 8<J_{2} / J_{1} \lesssim 0.4$ and a thermal phase transition into a magnetically ordered ground state for $J_{2} / J_{1} \gtrsim 0.4$. For the spin-1 system of interest in the context of $\mathrm{NiRh}_{2} \mathrm{O}_{4}$, these findings support the notion that quantum fluctuations paired with strong geometric frustration can indeed prevent the formation of magnetic ordering, and that the system remains fluctuating amongst different spin spiral states down to the zero temperature. However, when considering a slightly modified exchange model with two distinct types of next-nearest neighbor exchanges that has been proposed [19] for the tetragonal phase of $\mathrm{NiRh}_{2} \mathrm{O}_{4}$, we find that this picture no longer holds. In fact, we find that the modified energetics strongly inhibit the spin spiral fluctuations and instead favor the formation of conventional Néel order for arbitrary spin length $S$. We will return to this point towards the end of the manuscript and discuss how to possibly consolidate these findings with the experimental absence of a thermal phase transition.

Pseudofermion FRG.-To explore the exchange model (1) we employ the PF-FRG approach [20], which recasts the original spin degrees of freedom in terms of auxiliary
Abrikosov fermions and then applies the well-developed FRG approach of fermionic systems $[25,26]$. In the language of the original spin model, the PF-FRG approach amounts to a concurrent $1 / S$ and $1 / N$ expansion that allows us to faithfully capture conventionally ordered magnetic states (typically favored already in the large- $S$ limit of the expansion) and spin liquid states (favored in the alternate large- $N$ limit) and is known to become exact in the separate limits of large $S$ [24] and large $N$ [27,28]. With the computational effort scaling quadratically with system size $\mathcal{O}\left(N_{L}^{2}\right)$ and quartically with the number of frequencies $\mathcal{O}\left(N_{\omega}^{4}\right)$, there is a trade-off in choosing larger system sizes versus finer energy (temperature) resolution. With a focus on the finite-temperature ordering tendencies in the RG flow, we have opted in our numerical simulations for a very finely spaced frequency mesh of 144 frequencies (in a logarithmic spacing) and a system size of $L=10$ lattice bonds in every spatial direction (with a total of $N_{L}=981$ sites) resulting in a total number of 24,219,720 differential equations to be integrated for every choice of coupling parameters.

Phase diagram.-A common starting point for the analysis of a PF-FRG calculation is to plot the magnetic susceptibility as a function of frequency cutoff $\Lambda$ as shown in Fig. 2 for the exchange model (1) at fixed coupling $J_{2} /\left|J_{1}\right|=0.73$ (relevant to $\mathrm{NiRh}_{2} \mathrm{O}_{4}$ ) and varying spin length $S$. For small spins $S=1 / 2$ and $S=1$, the susceptibility follows a smooth trajectory down to the lowest temperature and there is no obvious breakdown of the RG flow, which is typically interpreted as the absence of any magnetic ordering transition. Contrarily, for spins $S=3 / 2$ and larger the RG flow exhibits a clear breakdown that signals the onset of magnetic order. In fact, what is only a kink in the flow at $S=3 / 2$ becomes a true divergence in the classical limit $(S=50)$. We note that the critical cutoff $\Lambda_{c}$ [29], at which the flow breaks down slightly, shifts towards larger values for increasing spin length

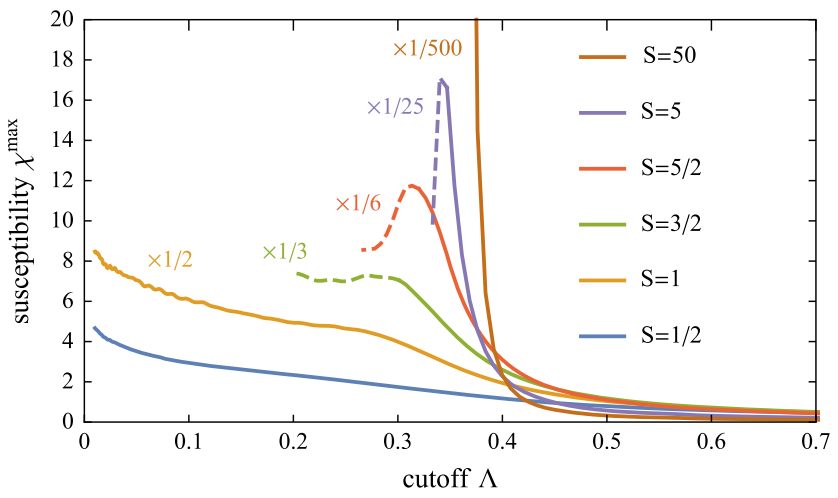

FIG. 2. Flow of susceptibility for different spin lengths $S$ for fixed couplings $J_{2} /\left|J_{1}\right|=0.73$. The energy scale is normalized by spin length and coupling strength, such that the flow breakdown occurs at similar scales. The susceptibility is always plotted at the momentum-space location where it is maximal. 


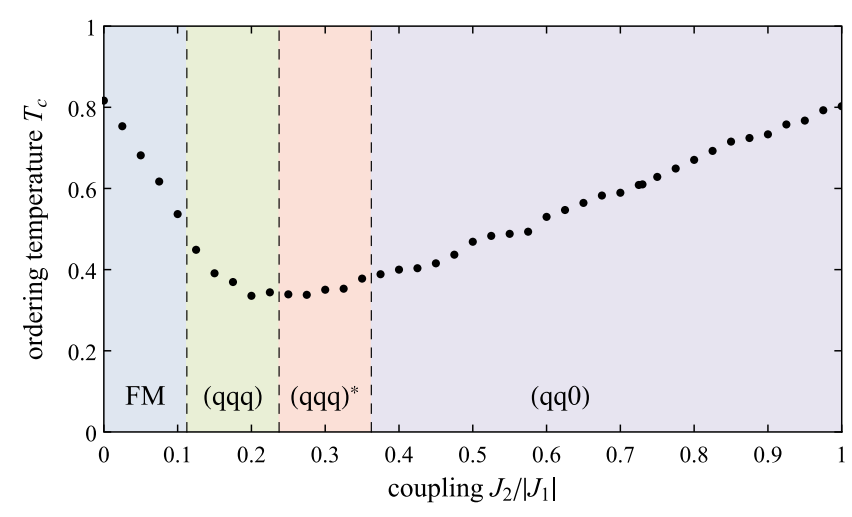

FIG. 3. Finite-temperature phase diagram. Shown is the transition temperature versus the coupling ratio $J_{2} /\left|J_{1}\right|$ for spin $S=5 / 2$. The background shadings indicates the different types of ground-state order, see the ground-state phase diagram of Fig. 6.

indicating a stronger ordering tendency as one approaches the classical limit.

Identifying the critical cutoff $\Lambda_{c}$ with a transition temperature $T_{c}=\Lambda_{c} \pi / 2$ [21,22], we can map out, for this classical regime, a finite-temperature phase diagram upon varying the ratio $J_{2} /\left|J_{1}\right|$ at fixed $J_{1}=-1$ [30], as illustrated for $S=5 / 2$ (relevant, e.g., to $\mathrm{MnSc}_{2} \mathrm{~S}_{4}$ ) in Fig. 3 . Similar to Monte Carlo results [11] for the classical exchange model, we find a significant suppression of the transition temperature for $1 / 8 \lesssim J_{2} /\left|J_{1}\right| \lesssim 0.4$, i.e., upon entering the spin spiral regime.

To explore the onset of magnetic ordering, it is highly instructive to track the evolution of the spin structure factor in the RG flow. This is illustrated for the spin-1 model in Fig. 4 below, where for fixed coupling $J_{2} /\left|J_{1}\right|=0.73$, we plot the top $20 \%$ of the spin structure factor and the color code reflects the relative strength - blue is low, red is high, and green is the top $0.4 \%$. For large cutoff $\Lambda$, the system fluctuates widely among many different possible magnetic orderings. In the low-temperature, small cutoff regime, however, we find that the features of the spin structure factor sharpen considerably and become highly reminiscent of the spin spiral surface found for the ground state of the classical $J_{1}-J_{2}$ exchange model [11]. This is visualized for the spin- 1 model for various values of the coupling ratio $J_{2} /\left|J_{1}\right|$ in Fig. 5 below. Ignoring the coloring scheme for a

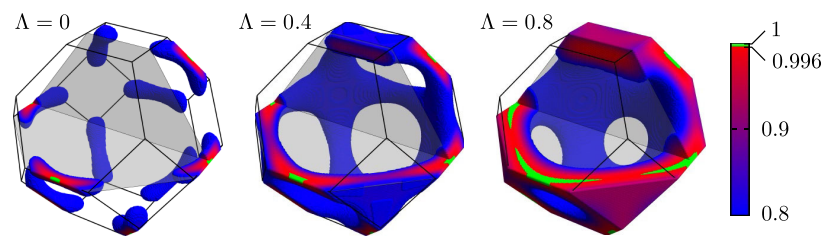

FIG. 4. Evolution of the spin structure factor with frequency cutoff $\Lambda$ for coupling $J_{2} /\left|J_{1}\right|=0.73$ and spin $S=1$. The colored regions mark the top $20 \%$ of the structure factor. Blue corresponds to $80 \%$ of the maximum value, red to $99.6 \%$. The top $0.4 \%$ are colored green. moment, one sees that the spin structure factor indeed retraces the spin spiral surface evolving from a spherical object for small $1 / 8<J_{2} /\left|J_{1}\right| \lesssim 0.2$ to an open surface that touches the border of the Brillouin zone and forms holes around the (qqq)-direction for larger $J_{2}$ to more linelike objects first around the $(q q 0)$ direction for $J_{2} /\left|J_{1}\right| \approx 1$ to two crossing linelike objects in the large $J_{2}$ limit. These observations fall in line with results for the spin structure factor of the classical exchange model obtained from Monte Carlo simulations [11]. Here, our focus is on further discerning the subset of points within the spiral surface where the structure factor is maximally enhanced, which provides an indicator of the magnetic ordering that will proliferate in case of a thermal phase transition and determine the ground state order. Tracking these points, one finds that beyond the Néel or ferromagnetic state for vanishing $J_{2}$, the preferred ordering momenta go for increasing $J_{2}$ through a sequence $(q q q) \rightarrow$ $(q q q)^{*} \rightarrow(q q 0) \rightarrow(q q 0)^{*} \rightarrow[q(q / 2) 0]$ (where the asterisk marks an ordering direction around a high-symmetry direction).

Repeating this analysis for varying spin length $S$ allows us to map out the general ground-state phase diagram of Fig. 6 as a function of both the coupling ratio $J_{2} /\left|J_{1}\right|$ and spin length $S$. We find that the general evolution of the spiral surface and the sequence of incipient ordering momenta do not change upon going from the quantum regime $(S=1 / 2)$ deep into the classical regime $(S=50)$ with only the boundary between the $(q q 0)$ and $(q q 0)^{*}$ order showing a noticeable dependence on the spin length $S$.

Quantum spiral spin liquids.-With the spin structure factor revealing the spiral surface, i.e., the manifold of approximately degenerate spin spirals at low temperatures, we can systematically investigate the effect of quantum fluctuations by varying the spin length $S$. In the classical limit $(S=50)$, the spiral surface determined via the spin structure factor indeed maps out a manifold of similar size and shape as found in the Luttinger-Tisza calculation $[31,32]$ for the ground state of the classical model (see Supplemental Material [33]). Increasing quantum fluctuations with decreasing spin length $S$, the spiral surfaces become not only more pronounced but systematically expand, similar to the trend observed for increasing the geometric frustration by ramping up $J_{2}$ in Fig. 5. This expansion can be readily explained by the fact that quantum systems gain more energy from antiferromagnetic fluctuations as opposed to ferromagnetic ones [34]. The absence of a thermal phase transition (see also Fig. 7 of the Supplemental Material [33]) for the low-spin systems with $S=1 / 2$ and $S=1$ points towards the formation of an unconventional ground state. In fact, the system remains fluctuating amongst different spin spiral states down to zero temperature. We dub this heavily fluctuating quantum state a quantum spiral spin liquid and note that this is a decisively different state from the topological paramagnet 

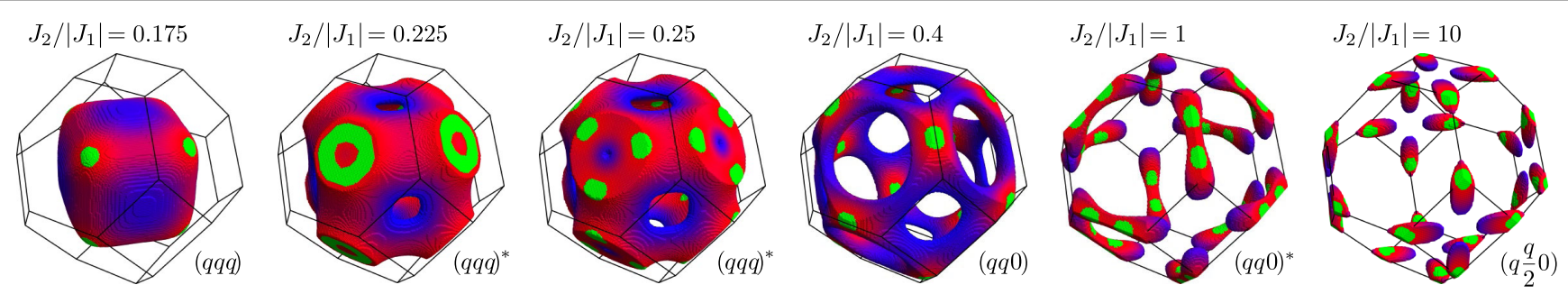

FIG. 5. Spin structure factor of the spin-1 model for varying coupling $\mathrm{J}_{2} /\left|\mathrm{J}_{1}\right|$. Depicted are the top $20 \%$ of the spin structure factor at frequency cutoff $\Lambda=0$ with the same color coding applied as in Fig. 4. The spin structure factor shows sharp surfacelike features whose evolution with $J_{2}$ reflects the spin spiral surface found in the ground state of the classical $J_{1}-J_{2}$ exchange model [11]. The maxima (indicated in green) describe a sequence of enhanced wave vectors (which characterize the onset of magnetic order for spins $S \geq 3 / 2$ ) at $(q q q) \rightarrow(q q q)^{*} \rightarrow(q q 0) \rightarrow(q q 0)^{*} \rightarrow[q(q / 2) 0]$ as $J_{2}$ is increased. Note that since the maximum of the structure factor is typically hidden inside the finite extent of the depicted manifold (see the right panel of Fig. 4 for an illustration) we project the maximum radially onto the surface of the manifold.

[35] recently suggested as ground state for the spin-1 model at hand.

$\mathrm{NiRh}_{2} \mathrm{O}_{4}$. - Let us finally turn to the spin- $1 A$-site spinel $\mathrm{NiRh}_{2} \mathrm{O}_{4}$ [19], whose recent synthesis has motivated the current study of the spin- $S J_{1}-J_{2}$ exchange model (1). $\mathrm{NiRh}_{2} \mathrm{O}_{4}$ exhibits strong antiferromagnetic couplings with a Curie-Weiss temperature of $\Theta_{\mathrm{CW}} \approx-10 \mathrm{~K}$ and shows no signs of a magnetic ordering transition down to $0.1 \mathrm{~K}$ [19]. While one might hope that this makes $\mathrm{NiRh}_{2} \mathrm{O}_{4}$ a prime candidate for the spin liquid physics of frustrated spin-1 diamond antiferromagnets discussed in this manuscript, there are some indications that the exchange model of Eq. (1) needs to be further expanded to truthfully capture the physics of $\mathrm{NiRh}_{2} \mathrm{O}_{4}$. For one, a structural transition of $\mathrm{NiRh}_{2} \mathrm{O}_{4}$ around $T \approx 400 \mathrm{~K}$ introduces a tetragonal distortion that requires to discriminate between in-plane $J_{2}^{-}$ and out of plane $J \frac{\perp}{2}$ next-nearest neighbor couplings (indicated in Fig. 1). Ab initio theory [19] suggests that the relevant coupling strengths for $\mathrm{NiRh}_{2} \mathrm{O}_{4}$ are given by $J_{1}=1, J_{2}^{-}=0.73, J_{2}^{\perp}=-0.91$ with antiferromagnetic $J_{1}$, $J_{2}^{-}$, and ferromagnetic $J_{2}^{\perp}$. If, however, we consider these

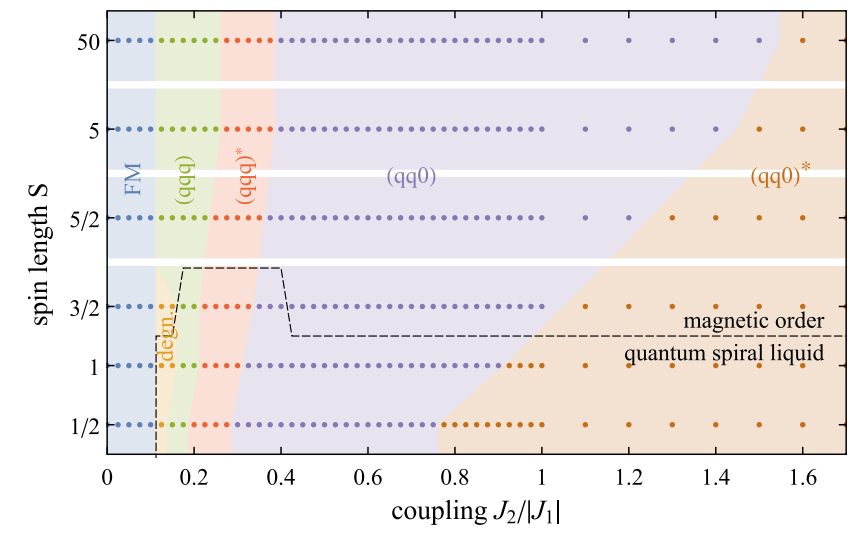

FIG. 6. Ground state phase diagram. The zero-temperature magnetic ordering (indicated by the coloring) as a function of the coupling ratio $J_{2} /\left|J_{1}\right|$ and spin lengths varying from the quantum limit $S=1 / 2$ (bottom) to the classical limit $S=50$ (top). two distinct types of next-nearest neighbor couplings, we find both in a Luttinger-Tisza calculation for the classical limit as well as in our PF-FRG calculations for all spin $S$, a conventional, Néel ordered ground state that is accompanied by a finite-temperature transition for arbitrarily small tetragonal splitting of the next-nearest neighbor interactions.

One possible way to defy this magnetic ordering tendency in the presence of a tetragonal distortion is to introduce a local single-ion spin anisotropy term $\sim D \sum_{i} S_{i}^{z} S_{i}^{z}$ as a novel source of frustration [36]. Indeed, we find in our PF-FRG calculations [37] that the latter stabilizes an extended paramagnetic phase where the system effectively decouples into single sites and thus exhibits a featureless spin structure factor as opposed to the quantum spiral spin liquid discussed above. For the original $J_{1}-J_{2}$ model, the spiral spin liquid gives way to a featureless paramagnetic regime around $D / J_{1} \approx 2$ (see Supplemental Material [33]), while the magnetic order in the presence of a tetragonal splitting is more robust and the critical value of the single ion anisotropy quickly rises [37]. The resulting phase diagram is displayed in Fig. 7, where

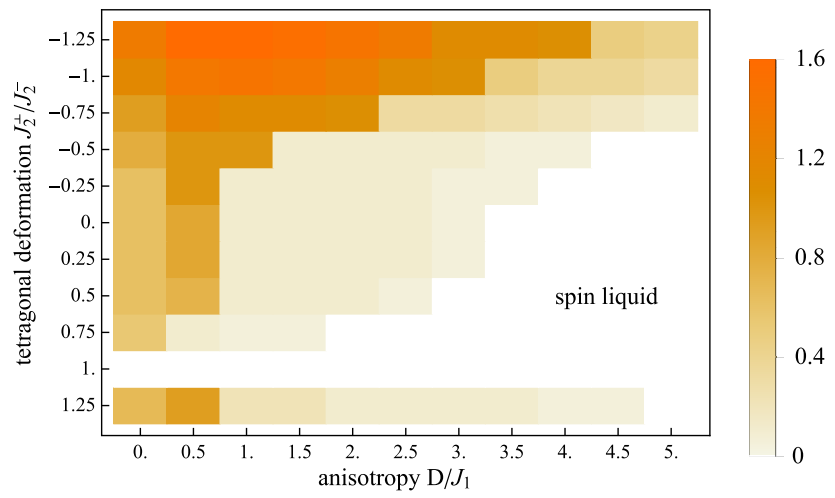

FIG. 7. Effects of a local spin-anisotropy on the tetragonally deformed diamond lattice. Displayed is the breakdown scale $\Lambda_{c}$ of the flow. The absence of a breakdown $\left(\Lambda_{c}=0\right)$ indicates the absence of magnetic order [38]. 
we plot the RG breakdown scale as a function of the strength of the tetragonal deformation and the local spin anisotropy $D / J_{1}$. The finite breakdown scale $\Lambda_{c}$ indicates magnetic order that is stabilized by a tetragonal deformation $J_{2}^{\perp} / J_{2}^{-} \neq 1$. For a finite spin anisotropy, however, an extended paramagnetic regime (corresponding to the white regime in Fig. 7 indicating a vanishing $\Lambda_{c}$ ) is stabilized.

While for the suggested $a b$ initio parameters for $\mathrm{NiRh}_{2} \mathrm{O}_{4}$ [19] (with $J_{2}^{\perp} / J_{2}^{-}=-1.25$ ) we do not see a transition into the paramagnet up to values of $D / J_{1} \approx 8$, already a relative small reduction of this coupling ratio (i.e., a smaller tetragonal splitting) would suffice to explain the absence of magnetic order observed in experiment. Looking ahead, it is thus desirable to compare our model calculations with extended $a b$ initio calculations that explicitly include the single-ion spin anisotropy. Experimentally, neutron diffraction experiments, such as the ones recently undertaken for $\mathrm{MnSc}_{2} \mathrm{~S}_{4}$ [16], could reveal whether the physics of $\mathrm{NiRh}_{2} \mathrm{O}_{4}$ is dominated by spin spiral liquid correlations or trivial paramagnetism.

We thank J. Attig for providing us with the LuttingerTisza result for the classical limit of the modified exchange model for $\mathrm{NiRh}_{2} \mathrm{O}_{4}$. This work was partially supported by the DFG within the CRC 1238 (project C02) and Transregio CRC 183 (project A02). The numerical simulations were performed on the CHEOPS cluster at RRZK Cologne and the JURECA cluster at the Forschungszentrum Juelich. J. R. is supported by the Freie Universität Berlin within the Excellence Initiative of the German Research Foundation. F. L. B. thanks the Bonn-Cologne Graduate School of Physics and Astronomy (BCGS) for support.

[1] H. Takagi and S. Niitaka, Highly frustrated magnetism in spinels, in Introduction to Frustrated Magnetism: Materials, Experiments, Theory, edited by C. Lacroix, P. Mendels, and F. Mila (Springer Berlin Heidelberg, Berlin, 2011), pp. $155-175$.

[2] L. Balents, Spin liquids in frustrated magnets, Nature (London) 464, 199 (2010).

[3] L. Savary and L. Balents, Quantum spin liquids: a review, Rep. Prog. Phys. 80, 016502 (2017).

[4] R. Moessner and J. T. Chalker, Properties of a Classical Spin Liquid: The Heisenberg Pyrochlore Antiferromagnet, Phys. Rev. Lett. 80, 2929 (1998).

[5] R. Moessner and J. T. Chalker, Low-temperature properties of classical geometrically frustrated antiferromagnets, Phys. Rev. B 58, 12049 (1998).

[6] B. Canals and C. Lacroix, Pyrochlore Antiferromagnet: A Three-Dimensional Quantum Spin Liquid, Phys. Rev. Lett. 80, 2933 (1998).

[7] B. Canals and C. Lacroix, Quantum spin liquid: The Heisenberg antiferromagnet on the three-dimensional pyrochlore lattice, Phys. Rev. B 61, 1149 (2000).

[8] V. Fritsch, J. Hemberger, N. Büttgen, E.-W. Scheidt, H.-A. Krug von Nidda, A. Loidl, and V. Tsurkan, Spin and Orbital
Frustration in $\mathrm{MnSc}_{2} \mathrm{~S}_{4}$ and $\mathrm{FeSc}_{2} \mathrm{~S}_{4}$, Phys. Rev. Lett. 92, 116401 (2004).

[9] N. Tristan, J. Hemberger, A. Krimmel, H.-A. Krug von Nidda, V. Tsurkan, and A. Loidl, Geometric frustration in the cubic spinels $M_{\mathrm{Al}_{2} \mathrm{O}_{4}}(M=\mathrm{Co}, \mathrm{Fe}$, and $\mathrm{Mn})$, Phys. Rev. B 72, 174404 (2005).

[10] T. Suzuki, H. Nagai, M. Nohara, and H. Takagi, Melting of antiferromagnetic ordering in spinel oxide $\mathrm{CoAl}_{2} \mathrm{O}_{4}, \mathrm{~J}$. Phys. Condens. Matter 19, 145265 (2007).

[11] D. Bergman, J. Alicea, E. Gull, S. Trebst, and L. Balents, Order-by-disorder and spiral spin-liquid in frustrated diamond-lattice antiferromagnets, Nat. Phys. 3, 487 (2007).

[12] J. Attig and S. Trebst, Classical spin spirals in frustrated magnets from free-fermion band topology, Phys. Rev. B 96, 085145 (2017).

[13] J.-S. Bernier, M. J. Lawler, and Y. B. Kim, Quantum Order by Disorder in Frustrated Diamond Lattice Antiferromagnets, Phys. Rev. Lett. 101, 047201 (2008).

[14] S. Lee and L. Balents, Theory of the ordered phase in $A$-site antiferromagnetic spinels, Phys. Rev. B 78, 144417 (2008).

[15] L. Savary, E. Gull, S. Trebst, J. Alicea, D. Bergman, and L. Balents, Impurity effects in highly frustrated diamondlattice antiferromagnets, Phys. Rev. B 84, 064438 (2011).

[16] S. Gao, O. Zaharko, V. Tsurkan, Y. Su, J. S. White, G. S. Tucker, B. Roessli, F. Bourdarot, R. Sibille, D. Chernyshov, T. Fennell, A. Loidl, and C. Ruegg, Spiral spin-liquid and the emergence of a vortex-like state in $\mathrm{MnSc}_{2} \mathrm{~S}_{4}$, Nat. Phys. 13, 157 (2017).

[17] G. Chen, L. Balents, and A. P. Schnyder, Spin-Orbital Singlet and Quantum Critical Point on the Diamond Lattice: $\mathrm{FeSc}_{2} \mathbf{S}_{4}$, Phys. Rev. Lett. 102, 096406 (2009).

[18] G. Chen, A. P. Schnyder, and L. Balents, Excitation spectrum and magnetic field effects in a quantum critical spinorbital system: The case of $\mathrm{FeSc}_{2} \mathrm{~S}_{4}$, Phys. Rev. B 80, 224409 (2009).

[19] J. R. Chamorro and T. M. McQueen, Frustrated S = 1 On A Diamond Lattice, arXiv:1701.06674.

[20] J. Reuther and P. Wölfle, $\mathrm{J}_{1}-\mathrm{J}_{2}$ frustrated two-dimensional Heisenberg model: Random phase approximation and functional renormalization group, Phys. Rev. B 81, 144410 (2010).

[21] Y. Iqbal, R. Thomale, F. Parisen Toldin, S. Rachel, and J. Reuther, Functional renormalization group for threedimensional quantum magnetism, Phys. Rev. B 94, 140408 (2016).

[22] F. L. Buessen and S. Trebst, Competing magnetic orders and spin liquids in two- and three-dimensional kagome systems: Pseudofermion functional renormalization group perspective, Phys. Rev. B 94, 235138 (2016).

[23] Y. Iqbal, T. Müller, K. Riedl, J. Reuther, S. Rachel, R. Valentí, M. J. P. Gingras, R. Thomale, and H. O. Jeschke, Signatures of a gearwheel quantum spin liquid in a spin- $-\frac{1}{2}$ pyrochlore molybdate Heisenberg antiferromagnet, Phys. Rev. Mater. 1, 071201 (2017).

[24] M. L. Baez and J. Reuther, Numerical treatment of spin systems with unrestricted spin length $S$ : A functional renormalization group study, Phys. Rev. B 96, 045144 (2017)

[25] C. Wetterich, Exact evolution equation for the effective potential, Phys. Lett. B 301, 90 (1993). 
[26] A. A. Katanin, Fulfillment of Ward identities in the functional renormalization group approach, Phys. Rev. B 70, 115109 (2004).

[27] F. L. Buessen, D. Roscher, S. Diehl, and S. Trebst, Functional renormalization group approach to SU(N) Heisenberg models: Real-space RG at arbitrary $\mathrm{N}$, arXiv:1711.02182.

[28] D. Roscher, F. L. Buessen, M. M. Scherer, S. Trebst, and S. Diehl, Functional renormalization group approach to SU(N) Heisenberg models: Momentum-space RG for the large-N limit, arXiv:1711.02183.

[29] Note that we have rescaled all energy scales in the manuscript by a factor of $2 S \sqrt{J_{1}^{2}+J_{2}^{2}}$.

[30] The choice of the ferromagnetic nearest neighbor coupling $J_{1}$ is primarily motivated to simplify the discussion of the structure factor data (by restricting it to the 1st Brillouin zone). In general, we find that the quantum system is, similarly to its classical counterpart, largely insensitive to the sign of the nearest neighbor coupling.

[31] J. M. Luttinger, A note on the ground state in antiferromagnetics, Phys. Rev. 81, 1015 (1951).

[32] J. M. Luttinger and L. Tisza, Theory of dipole interaction in crystals, Phys. Rev. 70, 954 (1946).
[33] See Supplemental Material at http://link.aps.org/ supplemental/10.1103/PhysRevLett.120.057201 for additional calculations and technical details on the PF-FRG implementation.

[34] This expansion of the spiral surface upon decreasing the spin length is also the reason for the shifts of the phase boundaries in the ground state phase diagram of Fig. 6.

[35] C. Wang, A. Nahum, and T. Senthil, Topological paramagnetism in frustrated spin-1 Mott insulators, Phys. Rev. B 91, 195131 (2015).

[36] G. Chen, Quantum paramagnet and frustrated quantum criticality in a spin-one diamond lattice antiferromagnet, Phys. Rev. B 96, 020412 (2017).

[37] For PF-FRG calculations including the spin anisotropy term, special care has to be taken to assure that the FRG flow remains within the spin-1 subspace (see Supplemental Material [33]). For the analysis of the phase diagram in Fig. 7, we fix the level repulsion term to $A / D=4$. This does not strictly constrain the system to the physical part of the Hilbert space but should suffice as a guideline for future work.

[38] Note that energy scales in the plot are renormalized by a factor $\sqrt{J_{1}^{2}+\left(J_{2}^{-}\right)^{2}+\left(J_{2}^{\perp}\right)^{2}+D^{2}}$. 